\title{
Evaluation of prognostic histological parameters proposed for pleural mesothelioma in diffuse malignant peritoneal mesothelioma. A short report
}

Federica Pezzuto ${ }^{1 *}$ D, Luigi Vimercati ${ }^{2}$, Francesco Fortarezza ${ }^{1}$, Andrea Marzullo ${ }^{3}$, Antonio Pennella ${ }^{4}$, Domenica Cavone ${ }^{2}$, Alessandra Punzi ${ }^{5}$, Concetta Caporusso ${ }^{3}$, Antonio d'Amati ${ }^{3}$, Teresa Lettini ${ }^{3}$ and Gabriella Serio ${ }^{3}$

\begin{abstract}
Introduction: Diffuse malignant peritoneal mesothelioma (DMPM) is a rare malignant neoplasm with poor survival that shares some similarities with the best-known pleural variant, pleural mesothelioma. The recent European Reference Network on Rare Adult Cancers (EURACAN)/International Association for the Study of Lung Cancer (IASL C) proposals attempted to improve the histological diagnosis and patient risk stratification. Herein, we investigated whether the pathology recommendations and suggestions of the pleural proposals were applicable to diffuse malignant peritoneal mesothelioma.

Methods: Fifty multiple laparoscopic biopsies of DMPM were consecutively collected at the Pathology Unit of the University of Bari. A two-tier system, i.e., low, and high grade, was used to categorize 34 epithelioid DMPMs. Architectural patterns, cytological features and stromal changes were also reported. Immunohistochemistry was performed for BRCA1-associated protein 1 (BAP1), programmed death-ligand 1 (PD-L1), and Ki67, while fluorescence in situ hybridization (FISH) was performed for p16/cyclin-dependent kinase inhibitor 2A (CDKN2A).

Results: High-grade epithelioid mesothelioma, high Ki67, and p16/CDKN2A deletion were significantly associated with short survival ( $p=0.004, p<0.0001$, and $p=0.002$, respectively). BAP1 loss and PD-L1 negativity were the most common findings. Multivariate analysis revealed that the nuclear grading system and p16 deletion significantly correlated with survival ( $p=0.003$ each).

Conclusions: The present study examined the prognostic significance of several factors proposed for pleural mesothelioma in an extra pleural site. Notably, the introduction of a grading system may provide better risk stratification in epithelioid DMPM. Ki67, BAP1 and p16/CDKN2A should also be measured whenever possible. A detailed report with all supportive data would allow us to collect sufficient information for use in further studies on larger case series.
\end{abstract}

Keywords: Pleural mesothelioma, Peritoneal mesothelioma, Histology, Grading

\footnotetext{
* Correspondence: federica.pezzuto@unipd.it

${ }^{1}$ Pathology Unit Department of Cardiac, Thoracic, Vascular Sciences and Public Health, University of Padova, via A. Gabelli 61, 35121 Padova, Italy Full list of author information is available at the end of the article
} 


\section{Introduction}

Malignant mesothelioma is a rare, fatal malignancy that primarily affects the visceral pleura. Most studies have been performed on the pleural variant, from which some information on the peritoneal form has been extrapolated. Most mesotheliomas are etiologically attributable to environmental asbestos exposure [1, 2], but a certain genetic susceptibility has also been investigated [3]. Although several differences have been found between the two entities, pleural and peritoneal mesotheliomas share some similarities. Diffuse malignant peritoneal mesothelioma (DMPM) has a poor prognosis. Longterm survivors are rare and exceptionally described in the literature [4]. The median survival is shorter than 1 year if untreated, but treatment strategies are limited. Cytoreductive surgery and heated intraperitoneal chemotherapy are the primary therapies for resectable forms [5]. Because the advantages obtained in prognosis and treatment response remain limited, the development of sensitive/specific diagnostic systems and the identification of prognostic/predictive factors are a priority. Some recommendations for appropriate pathology reports and diagnoses in DMPM have been established recently [5]. Some histological and immunohistochemical factors have also been investigated as prognostic indicators [6]. The recent European Reference Network on Rare Adult Cancers (EURACAN)/International Association for the Study of Lung Cancer (IASLC) proposal [7] was a step toward a multidisciplinary approach to pleural mesothelioma to improve the diagnostic precision and patient risk stratification. We investigated whether the pathology recommendations and suggestions of the pleural proposal were applicable to DMPM. In particular, we focused on histological parameters with a prognostic implication in a broad sense that is histotype, prevalent architectural pattern, cytological and stromal features, nuclear grading, necrosis, BAP-1 expression and PD-L1 positivity. In addition, the proliferative index has also been considered, as strongly recommended by the last Peritoneal Surface Oncology Group International (PSOGI)/EURACAN clinical practice guidelines for DMPM.

\section{Materials and methods}

The histological diagnosis of DMPM was confirmed on 50 multiple laparoscopic biopsies that were consecutively collected at the Pathology Unit of the University of Bari between March 1990 and December 2017. A single sample was evaluated for each patient. When multiple specimens were available, the most representative sample (in terms of neoplastic cell content) was chosen.

The local Ethics Committee of the Policlinic Hospital, Bari, Italy approved the study. A panel of antibodies was used for diagnosis [5]. Samples were classified as epithelioid, biphasic and sarcomatoid histological subtypes. The epithelioid variant was further evaluated for predominant architectural patterns (tubulopapillary, trabecular, adenomatoid, microcystic, solid, micropapillary, transitional, pleomorphic, when detected in greater than $50 \%$ of the tumor surface), cytological features (rhabdoid, deciduoid, small cell, clear cell, signet ring, lymphohistiocytoid), stromal characteristics (myxoid), nuclear atypia, mitotic count and the presence of necrosis [8]. Nuclear atypia was scored from 1 (mild) to 3 (severe). Mitotic count was assigned a score of 1, 2 or 3 based on mitoses in $1,2-4$ and $\geq 5$ per $2 \mathrm{~mm}^{2}$, respectively. The nuclear grade was derived from the sum of the scores for atypia and mitotic count: sum of 2-3 was grade I; sum of 4-5 was grade II, and sum of 6 was grade III. Tumours were further categorized into low-grade tumors (tumors with nuclear grade I and nuclear grade II without necrosis) and high-grade tumors (tumors with nuclear grade II in the presence of necrosis and nuclear grade III).

Immunohistochemistry for Ki67 (MIB-1, clone K5001, DAKO) and BRCA1-associated protein 1 (BAP1) (C4, Santa Cruz Biotechnology, Santa Cruz, USA) was performed on all samples. Ki67 expression was expressed as the percentage of positive cells in the total cell number. A complete absence of nuclear staining was considered true negative BAP1 staining in the presence of nuclearpositive lymphocytes. Programmed death-ligand 1 (PD-L1) (clone 22C3, DAKO) was examined in 27 cases. P16/cyclin-dependent kinase inhibitor 2A (CDKN2A) deletion was also assessed using fluorescence in situ hybridization (FISH) analysis, as previously described [6].

Univariate analyses were performed using the nonparametric Mann-Whitney U or Kruskal-Wallis tests for continuous variables and Fisher's exact test or chisquared test for categorical variables. Intra-observer and interobserver data reproducibility were tested for mitotic count, Ki67 value and tumor grade by considering all cases recorded by two observers (FP and GS), one of whom recorded the series twice at different times. The observer variations and correlations were examined using paired t tests and Pearson's correlation coefficient.

Survival was calculated from the day of the pathological diagnosis to death or last follow-up. The overall survival was calculated using the Kaplan-Meier method with log-rank analysis. Multiple linear regression analyses were also performed. Significance was two-tailed and set at 0.05 .

\section{Results}

Fifty patients with a diagnosis of DMPM were included. The median age was 63 years (mean $63.8 \pm 11.5$; range 36-89 years). Thirteen patients were females (26\%). Our series was quite homogeneous in treatment, although the data were collected over a long-time frame. All the 
Table. 1 Histologic subtypes, immunohistochemical and molecular features of diffuse malignant peritoneal mesothelioma

\begin{tabular}{|c|c|}
\hline \multicolumn{2}{|c|}{ Histological subtypes [N (\%)] } \\
\hline - Epithelioid & $34(68 \%)$ \\
\hline - Biphasic & $12(24 \%)$ \\
\hline - Sarcomatoid & $4(8 \%)$ \\
\hline \multicolumn{2}{|l|}{ CDKN2A status [N (\%)] } \\
\hline - Homozygous deletion & $30(60 \%)$ \\
\hline - Heterozygous deletion & $12(24 \%)$ \\
\hline - No deletion & $8(15 \%)$ \\
\hline BAP1 loss [N (\%)] & $43(86 \%)$ \\
\hline \multicolumn{2}{|c|}{ PD-L1 (tumor proportion score) [N (\%)] } \\
\hline$-\quad<1 \%$ & $40(98 \%)$ \\
\hline$-\quad 1-49 \%$ & $1(2 \%)$ \\
\hline$-\quad \geq 50 \%$ & 0 \\
\hline Ki67 [\%, median (Q1-Q3)] & $25.5(15.25-41)$ \\
\hline
\end{tabular}

patients were chemo-naïve at the time of biopsy. None of the patients underwent cytoreductive surgical therapy, intraperitoneal chemotherapy or immunotherapy after diagnosis. All patients had died at the last follow-up. There were 34 cases (68\%) of epithelioid, 12 (24\%) biphasic and 4 (8\%) sarcomatoid histological subtypes (Table 1). A predominantly solid architectural pattern was most frequent in epithelioid mesotheliomas, and it was present in $25(73 \%)$ cases. Six cases (18\%) showed a desmoplastic stromal reaction, and $1(3 \%)$ case showed myxoid changes. Nuclear atypia 2 and 3 were present in 16 and 18 cases, respectively. The median mitotic count was 5 per $2 \mathrm{~mm}^{2}$ (mean $6.4 \pm 5.3$; range $1-29$ ). Foci of necrosis were evident in $12(35.3 \%)$ cases. Epithelioid mesotheliomas were classified into low grade (Fig. 1a, b) and high grade (Fig. 1c, d) and detected in 15 and 19 cases, respectively (Table 2). The median percentage of Ki67 was $25.5 \%$. BAP1 loss was found in 43 cases (86\%). PD-L1 was positive in one (2\%) biphasic case. The p16/ $C D K N 2 A$ homozygous deletion was detected in $30(60 \%)$ cases (Table 1). High-grade tumors showed significantly different Ki67 values than low-grade tumors (mean $28.9 \pm 12,8$, CI $95 \%$ 22.7-35.1; median 26 vs. mean $16.1 \pm 13.7$, CI 95\% 8.5-23.7, median 13.5; $p=0.009$ ). For the reproducibility test, the $t$ test was not significant in all cases. The correlation coefficient was $r=.987$ for the interobserver comparison of Ki67, and this coefficient was significant $(p=0.03)$.

The median survival was 6 months (Q1-Q3: 3-14; mean $13.7 \pm 16.9$; CI 95\% 9.04-18.4; range 1-60). Survival showed statistical significance for sex, and it was lower in females. A significant difference in survival was detected according to the histological subtypes, with the shortest survival observed in the sarcomatoid $(p=$ $0.002)$ mesotheliomas, and in high-grade epithelioid tumors $(p=0.004)$ (Fig. 1e). A low Ki67 percentage, nuclear grade II, no deletion/heterozygous deletion of $p 16 /$ $C D K N 2 A$ showed the longest survival $(p=0.004, p=$ 0.0001 and $p=0.002$, respectively). No statistical significance was found for BAP1 loss, even when a longer

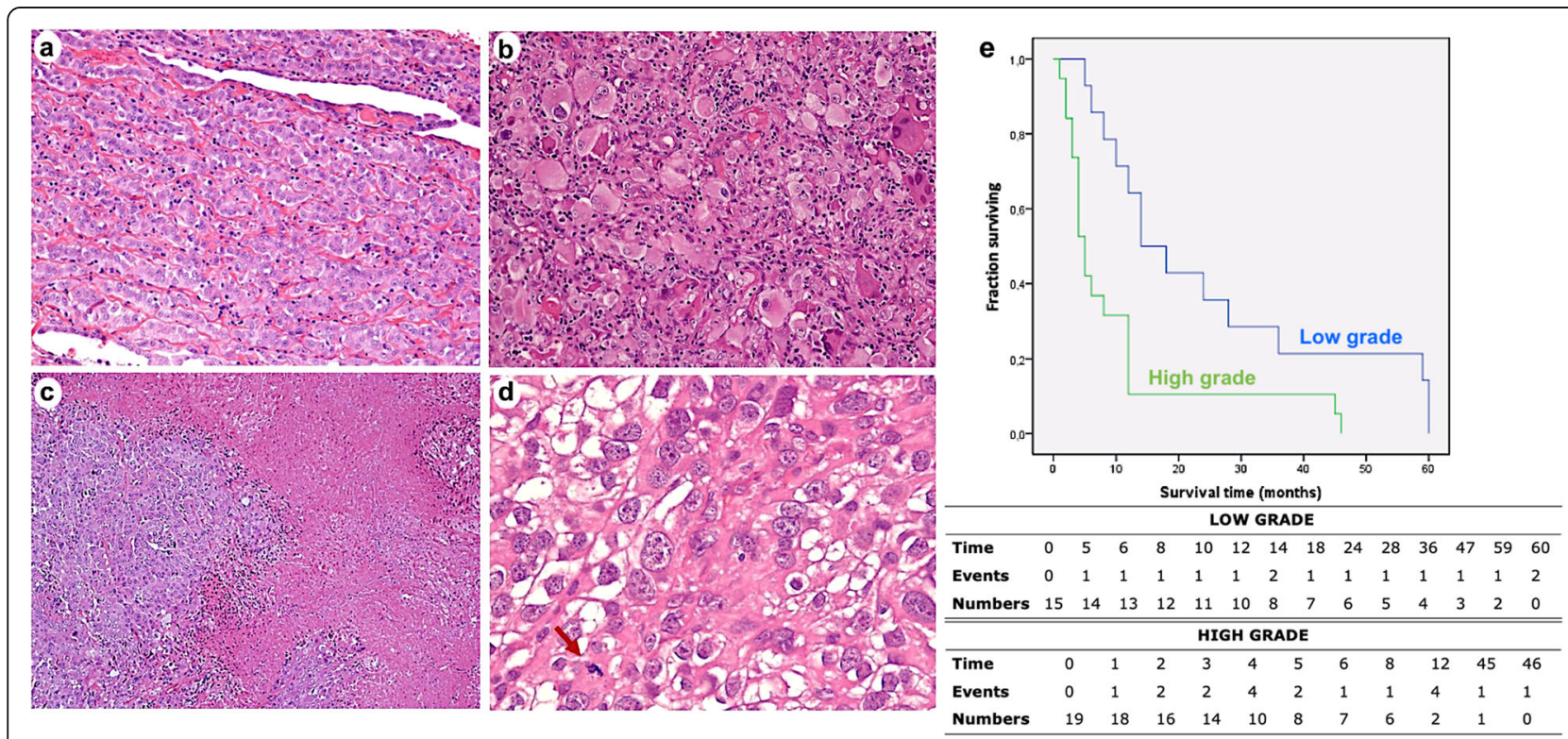

Fig. 1 Low- and high-grade tumors. Representative images of low- (a, hematoxylin and eosin stain, original magnification $\times 100)$ and high-grade tumors with marked nuclear atypia (b, hematoxylin and eosin stain, original magnification $\times 200$ ), foci of necrosis (c), hematoxylin and eosin stain, original magnification $\times 100)$ and high mitotic count $(\mathbf{d}$, hematoxylin and eosin stain, original magnification $\times 400)$. A significant difference in survival was detected $(p=0.004)$ according to the tumor grade $(\mathbf{e})$ 
Table. 2 Morphological features of diffuse malignant epithelioid peritoneal mesothelioma

\begin{tabular}{|c|c|}
\hline Architectural pattern & N (\%) \\
\hline - Tubulopapillary & $6(18 \%)$ \\
\hline - Trabecular & $1(3 \%)$ \\
\hline - Microcystic & $1(3 \%)$ \\
\hline - Solid & $25(73 \%)$ \\
\hline - Micropapillary & $1(3 \%)$ \\
\hline \multicolumn{2}{|l|}{ Cytological features } \\
\hline - Epithelioid & $28(82 \%)$ \\
\hline - Deciduoid & $6(18 \%)$ \\
\hline \multicolumn{2}{|l|}{ Stromal features } \\
\hline - Non reactive & 27 (79\%) \\
\hline - Desmoplastic & $6(18 \%)$ \\
\hline - Myxoid & $1(3 \%)$ \\
\hline \multicolumn{2}{|l|}{ Nuclear atypia } \\
\hline-1 & 0 \\
\hline 2 & $16(47 \%)$ \\
\hline-3 & $18(53 \%)$ \\
\hline \multicolumn{2}{|l|}{ Mitotic count } \\
\hline$-\quad 1\left(\leq 1 / 2 \mathrm{~mm}^{2}\right)$ & $1(3 \%)$ \\
\hline$-\quad 2\left(2-4 / 2 \mathrm{~mm}^{2}\right)$ & $15(44 \%)$ \\
\hline$-\quad 3\left(\geq 5 / 2 \mathrm{~mm}^{2}\right)$ & $18(53 \%)$ \\
\hline \multicolumn{2}{|l|}{ Necrosis } \\
\hline - Present & $12(35 \%)$ \\
\hline - Absent & $22(65 \%)$ \\
\hline \multicolumn{2}{|l|}{ Grade } \\
\hline - Low & 15 (44\%) \\
\hline - High & $19(56 \%)$ \\
\hline
\end{tabular}

median survival was detected for cases with BAP1 alteration (8 months vs. 2 months). The small number of cases with each architectural pattern and stromal change did not allow reliable statistical correlations to be drawn. Survival data are reported in Table 3.

Multiple linear regression analysis including Ki67 (both punctual values and cut-offs), nuclear grading, necrosis, and $p 16 / C D K N 2 A$ deletion showed that only nuclear grading and $p 16 / C D K N 2 A$ were significant predictors of survival ( $p=0.003$ each) (Table 4).

\section{Discussion}

The present study found that the grading system proposed for pleural malignant mesothelioma (MM) also significantly correlated with patient survival in DMPM. Several pathological grading systems have been proposed for pleural mesothelioma over the last decade [8-10]. However, none of these systems were standardized for DMPM. Valente et al. was the first study to combine nuclear features and mitoses for epithelioid DMPM [11] and showed a strong correlation with survival. A composite nuclear-grading system was successfully applied in a recent multi-institutional series of 225 cases [12]. Our results are consistent with this last study on the role of necrosis. Necrosis did not affect survival in our epithelioid case series when included in multivariate analysis. Our findings support the importance of establishing a standardized grading system to achieve better risk stratification in epithelioid DMPM. The application of the same method for all mesotheliomas, regardless of their origin, would be a step toward greater homogeneity in histological reports.

Other histological findings were associated with a distinct clinical outcome in our case series. Patients with epithelioid tumors had significantly longer survival than patients with other histological types. This result was not an unexpected finding because the current histological classification is a strong predictor of survival, with epithelioid MM having the best prognosis [13-15]. The epithelioid type was also the most frequently detected in our study population. This result is consistent with the literature [16]. Epithelioid MMs include a wide spectrum of tumors with variegated features that are associated with different clinical courses [7]. The clinical and pathological heterogeneity must be further characterized to identify pathological factors that may be associated with a more indolent tumor type or a poor outcome. The prognostic importance of architectural patterns and stromal reaction in DMPM are recognized [17]. even if concerns of the reproducibility of some histological parameters were raised [18]. We characterized the architectural and stromal features within our study population. However, our samples lacked some subgroups. Therefore, the evaluation of interobserver agreement and a comparison of survival were difficult to achieve, and evaluation should be postponed until a greater number of cases is available.

BAP1 expression was lost in most patients in our cohort. The complete loss of BAP1 expression was reported in a high percentage of MM [19], but never in reactive proliferation, which suggests the use of BAP1 as a highly specific method for differentiating MM and benign mesothelial proliferation [20]. BAP1 is only occasionally altered $(0.3 \%)$ in serous ovary carcinoma, which further supports its utility in supporting a pathological diagnosis of peritoneal mesothelioma rather than gynecological carcinoma [21]. BAP1 inactivation altered the clinical outcome [19]. Protein nuclear expression is extremely feasible and may be considered a reliable marker for the complete loss of BAP1 activity [19]. BAP1 was only detected in 7 cases in our study population, which limited the power of statistical tests. Because the immunohistochemical evaluation of BAP1 is useful 
Table. 3 Survival data

\begin{tabular}{|c|c|c|c|c|c|c|c|c|c|}
\hline \multirow[t]{3}{*}{ Parameter } & \multicolumn{4}{|c|}{ Mean survival (months) } & \multicolumn{4}{|c|}{ Median survival (months) } & \multirow[t]{3}{*}{$P$-Value } \\
\hline & \multicolumn{4}{|c|}{$\mathrm{Cl} 95 \%$} & \multicolumn{4}{|c|}{$\mathrm{Cl} 95 \%$} & \\
\hline & Estimate & SE & Lower limit & Higher limit & Estimate & SE & Lower limit & Higher limit & \\
\hline \multicolumn{10}{|l|}{ Sex } \\
\hline Males & 16.2 & 3.1 & 10.2 & 22.1 & 8 & 3 & 2.1 & 13.9 & $0.04^{*}$ \\
\hline Females & 8.8 & 2.1 & 2.7 & 10.8 & 3 & 1.8 & 0 & 6.6 & \\
\hline \multicolumn{10}{|l|}{ Age } \\
\hline$<63 \mathrm{yrs}$ & 11.4 & 3.3 & 5 & 17.8 & 5 & 1.5 & 2.1 & 7.9 & 0.58 \\
\hline$\geq 63$ yrs & 16.1 & 3.5 & 9.2 & 22.9 & 12 & 2.7 & 6.7 & 17.3 & \\
\hline \multicolumn{10}{|l|}{ Histotype } \\
\hline Epithelioid & 17.4 & 3.2 & 11.2 & 23.6 & 10 & 2.2 & 5.7 & 14.3 & \\
\hline Biphasic & 7 & 3 & 1.2 & 12.8 & 2 & 0.6 & 0.9 & 3.1 & $0.002^{*}$ \\
\hline Sarcomatoid & 2.8 & 1 & 0.7 & 4.8 & 1 & & & & \\
\hline \multicolumn{10}{|l|}{ Tumor grading } \\
\hline Low & 25.3 & 5.5 & 14.5 & 36 & 14 & 3.7 & 6.7 & 21.3 & $0.004^{*}$ \\
\hline High & 10 & 3 & 4.1 & 15.9 & 5 & 0.7 & 3.6 & 6.4 & \\
\hline \multicolumn{10}{|l|}{ Nuclear grading } \\
\hline 2 & 27.8 & 5.2 & 17.6 & 37.9 & 18 & 10 & 0 & 37.6 & $<0.0001^{*}$ \\
\hline 3 & 8.2 & 2.3 & 3.6 & 12.7 & 5 & 0.7 & 3.6 & 6.4 & \\
\hline \multicolumn{10}{|l|}{ Ki67 } \\
\hline Low & 24.1 & 4.3 & 15.8 & 32.5 & 14 & 2.7 & 8.8 & 19.2 & \\
\hline High & 5.5 & 1.3 & 2.9 & 8.1 & 4 & 0.7 & 2.6 & 5.4 & $<0.0001^{*}$ \\
\hline \multicolumn{10}{|l|}{ p16/CDKN2A } \\
\hline No deletion & 22 & 8.8 & 4.7 & 39.3 & 6 & 6.4 & 0 & 18.5 & \\
\hline Heterozygous & 23.7 & 5.5 & 12.9 & 34.3 & 12 & 2.7 & 6.8 & 17.3 & $0.002^{*}$ \\
\hline Homozygous & 7 & 1.5 & 4.1 & 10 & 4 & 0.9 & 2.3 & 5.8 & \\
\hline
\end{tabular}

Abbreviations: Cl Confidence interval; SE Standard error *for statistical significance

for diagnostic purposes and fairly specific for the diagnosis of MM in the appropriate histological context, it may be easily evaluated and inserted in the final report. Larger case series may be available in the near future to examine the prognostic significance.

As recommended for inclusion in pathological reports of pleural mesotheliomas, we also analyzed p16/ $C D K N 2 A$ deletion and PD-L1 status. The results of FISH analysis for $p 16 / C D K N 2 A$ showed the worst prognosis when a homozygous deletion was detected. This finding confirms our previous work [6] and is consistent with the literature. The prognostic significance of p16/ CDKN2A deletion in DMPM is well recognized [22], and it was suggested as a tool to help identify patients with a favorable outcome after multimodal treatments [23].

Positive PD-L1 immunostaining was detected in only one biphasic mesothelioma. PD-L1 tumor expression in DMPM was amply reported in the literature. One

Table. 4 Multiple linear regression analysis

\begin{tabular}{|c|c|c|c|c|c|c|c|c|c|c|c|c|}
\hline \multirow[t]{2}{*}{ Parameter } & \multicolumn{4}{|c|}{ Coefficient Table Iteration 1} & \multicolumn{4}{|c|}{ Coefficient Table Iteration 2} & \multicolumn{4}{|c|}{ Coefficient Table Iteration 3} \\
\hline & Coeff & SE & Stand Coeff & $P$-value & Coeff & SE & Stand Coeff & $P$-value & Coeff & SE & Stand Coeff & $P$-value \\
\hline Nuclear grading & -10.34 & 5.5 & -0.32 & 0.07 & -9.46 & 5.23 & -0.29 & 0.08 & -14.38 & 12.28 & -0.44 & $0.003^{*}$ \\
\hline Necrosis & 3.15 & 5.34 & 0.08 & 0.56 & - & - & - & - & - & - & - & - \\
\hline Ki67 (low/high) & -9.04 & 6.23 & -0.24 & 0.15 & -9.98 & 5.95 & -0.27 & 0.10 & - & - & - & - \\
\hline P16/CDKN2Adeletion & -10.4 & 3.28 & -0.43 & 0.004 & -9.96 & 3.16 & -0.04 & 0.004 & -10.51 & 3.23 & -0.44 & $0.003^{*}$ \\
\hline
\end{tabular}

Abbreviations: Coeff Coefficient, SE Standard error, Stand Standard 
plausible explanation for this discrepancy is the small sample size of non-epithelioid cases, which are more commonly PD-L1 positive [24, 25]. This result is important and warrants in-depth investigation.

The contribution of Ki67 was introduced as a strong recommendation in the last PSOGI/EURACAN clinical practice guidelines for DMPM [7]. Ki67 was not strictly associated with survival of epithelioid mesotheliomas in our multivariate analysis. This topic remains controversial for several reasons. Although its prognostic value was suggested in the literature, there is no consensus on which scoring system should be used [12, 13, 26-28]. A two-tier system of categorization for Ki67 may be informative for prognosis as punctual values, but standardization of the methodology is mandatory. Digital imaging and virtual scoring may be helpful for this purpose. Its role deserves further study due to the uncertainty of the results.

Females showed lower overall survival than males in our study. This result contrasts with previous works [29] but it may be due to the limited number of female patients recruited. None of our cases were treated with combined-modality management, but complete cytoreduction, lack of lymph node metastasis, and low peritoneal cancer index (PCI) [13, 30-33] strictly correlated with significant improvement in survival in DMPM. An evaluation of histological parameters could further improve these results via better patient risk stratification.

The present study has some limitations. First, it was a retrospective study design. The relatively small sample size did not allow all morphological evaluations and statistical correlations. Second, PD-L1 was not available for all patients due to the lack of neoplastic cells in the residual tissue. However, the high number of epithelioid cases allowed a reliable evaluation of the feasibility of the grading categorization.

This study examined the prognostic significance of factors proposed for pleural mesothelioma in an extrapleural site. However, these results must be seen from a specific peritoneal perspective. The two entities are distinct but some histological features could provide a help in terms of morphological prognosis (in particular the nuclear grading system) and could provide a reliable standard for the diagnostic reporting. The collection of sufficient information for use in further multicentric studies would allow the prognostic value of each factor to be determined and validated, and answer unsolved questions to orient patient treatment in the right direction. A detailed report specifying additional morphological, immunohistochemical and molecular supportive data is desirable in this rare disease.

\section{Abbreviations}

DMPM: Diffuse malignant peritoneal mesothelioma; MM: Malignant mesothelioma; EURACAN: European Reference Network on Rare Adult Cancers; IASLC: International Association for the Study of Lung Cancer;
WT1: Wilms' Tumor 1; BAP1: BRCA1 associated protein 1; PD-L1: Programmed Death-Ligand 1; FISH: fluorescence in situ hybridization

Acknowledgments

We thank Pragnell M. V, B.A., for language editing.

\section{Authors' contributions}

Gabriella Serio, Federica Pezzuto: conceptualization, manuscript writing, reviewing and editing, supervision. Francesco Fortarezza: writing original draft preparation, visualization, investigation; Andrea Marzullo, Antonio Pennella, Alessandra Punzi: resources, investigation, visualization; Concetta Caporusso, Domenica Cavone, Teresa Lettini, Antonio D'Amati: resources, investigation; Luigi Vimercati: conceptualization, manuscript writing, reviewing and editing, supervision. The authors read and approved the final manuscript.

\section{Funding}

This research did not receive any specific grant from public or commercial funding agencies.

\section{Availability of data and materials}

The datasets used andanalyzed during the current study are available from the corresponding author on reasonable request.

\section{Declarations}

Ethics approval and consent to participate

The local Ethics Committee of the Policlinic Hospital, Bari, Italy approved the study (accession number 5062, June 22, 2016).

Consent for publication

Not applicable.

\section{Competing interests}

The authors declare no competing interests.

\section{Author details}

'Pathology Unit Department of Cardiac, Thoracic, Vascular Sciences and Public Health, University of Padova, via A. Gabelli 61, 35121 Padova, Italy. ${ }^{2}$ Department of Interdisciplinary Medicine, Occupational Health Division, University of Bari, 1 Umberto I Sq. 70121 Bari, Italy. ${ }^{3}$ Pathology Unit,

Department of Emergency and Organ Transplantation - DETO, University of Bari, 1 Umberto I Sq., 70121 Bari, Italy. ${ }^{4}$ Pathology Unit, Department of Surgery, University of Foggia, 121 Napoli St, 71122 Foggia, Italy. ${ }^{5}$ Pathology Unit, IRCCS National Cancer Institute "Giovanni Paolo II", 65 Orazio Flacco St, 70124 Bari, Italy.

Received: 29 October 2020 Accepted: 6 July 2021

Published online: 22 July 2021

\section{References}

1. Vimercati L, Cavone D, Lovreglio P, et al. Environmental asbestos exposure and mesothelioma cases in Bari, Apulia region, southern Italy: a national interest site for land reclamation. Environ Sci Pollut Res Int. 2018;25:15692701. https://doi.org/10.1007/s11356-018-1618-x.

2. Serio G, Pagliarulo V, Marzullo A, Punzi A, Pezzuto F, Gentile M, et al. Molecular changes of malignant mesothelioma in the testis and their impact on prognosis: analyses of two cases. Int J Exp Pathol. 2016;9: 7658-67.

3. Serio G, Vimercati L, Pennella A, et al. Genomic changes of chromosomes 8p23.1 and 1q21: novel mutations in malignant mesothelioma. Lung Cancer. 2018;126:106-11. https://doi.org/10.1016/j.lungcan.2018.10.012.

4. Serio G, Pezzuto F, Marzullo A, et al. Peritoneal mesothelioma with residential asbestos exposure. Report of a case with long survival (seventeen years) analyzed by cgh-array. Int J Mol Sci. 2017;18:1818. Published 2017 Aug 22. https://doi.org/10.3390/ijms18081818.

5. Kusamura S, Kepenekian V, Villeneuve $L$, et al. Peritoneal mesothelioma: PSOGI/EURACAN clinical practice guidelines for diagnosis, treatment and follow-up [published online ahead of print, 2020 Mar 12]. Eur J Surg Oncol. 2020;S0748-7983(20)30113-X. https://doi.org/10.1016/j.ejso.2020.02.011. 
6. Pezzuto F, Serio G, Fortarezza F, et al. Prognostic Value of Ki67 percentage, WT-1 expression and p16/CDKN2A deletion in diffuse malignant peritoneal mesothelioma: a single-centre cohort study. Diagnostics (Basel). 2020;10: E386. Published 2020 Jun 9. https://doi.org/10.3390/diagnostics10060386.

7. Nicholson AG, Sauter JL, Nowak AK, et al. EURACAN/IASLC proposals for updating the histologic classification of pleural mesothelioma: towards a more multidisciplinary approach. J Thorac Oncol. 2020;15:29-49. https://doi. org/10.1016/j.jtho.2019.08.2506.

8. Rosen LE, Karrison T, Ananthanarayanan V, et al. Nuclear grade and necrosis predict prognosis in malignant epithelioid pleural mesothelioma: a multiinstitutional study. Mod Pathol. 2018;31:598-606. https://doi.org/10.1038/ modpathol.2017.170

9. Kadota K, Suzuki K, Colovos C, et al. A nuclear grading system is a strong predictor of survival in epitheloid diffuse malignant pleural mesothelioma. Mod Pathol. 2012;25:260-71. https://doi.org/10.1038/modpathol.2011.146.

10. Pelosi G, Papotti M, Righi L, et al. Pathologic grading of malignant pleural mesothelioma: an evidence-based proposal. J Thorac Oncol. 2018;13:175061. https://doi.org/10.1016/j.jtho.2018.07.002.

11. Valente $\mathrm{K}$, Blackham AU, Levine $\mathrm{E}$, et al. A Histomorphologic grading system that predicts overall survival in diffuse malignant peritoneal mesothelioma with epithelioid subtype. Am J Surg Pathol. 2016;40:1243-8. https://doi. org/10.1097/PAS.0000000000000696.

12. Chapel DB, Schulte JJ, Absenger G, Attanoos R, et al. Malignant peritoneal mesothelioma: prognostic significance of clinical and pathologic parameters and validation of a nuclear-grading system in a multi-institutional series of 225 cases. Mod Pathol. 2020. https://doi.org/10.1038/s41379-020-00688-4.

13. Yan TD, Deraco M, Baratti D, et al. Cytoreductive surgery and hyperthermic intraperitoneal chemotherapy for malignant peritoneal mesothelioma: multi-institutional experience. J Clin Oncol. 2009;27:6237-42. https://doi. org/10.1200/JCO.2009.23.9640.

14. Liu S, Staats $P$, Lee $M$, et al. Diffuse mesothelioma of the peritoneum: correlation between histological and clinical parameters and survival in 73 patients. Pathology. 2014;46:604-9. https://doi.org/10.1097/PAT. 0000000000000181.

15. Votanopoulos Kl, Sugarbaker P, Deraco M, et al. Is Cytoreductive surgery with Hyperthermic Intraperitoneal chemotherapy justified for biphasic variants of peritoneal mesothelioma? Outcomes from the peritoneal surface oncology group international registry. Ann Surg Oncol. 2018;25:667-73. https://doi.org/10.1245/s10434-017-6293-5.

16. Galateau-Salle F, Churg A, Roggli V, et al. The 2015 World Health Organization classification of tumors of the pleura: advances since the 2004 classification. J Thorac Oncol. 2016;11:142-54. https://doi.org/10.1016/j.jtho.2 015.11.005.

17. Krasinskas AM, Borczuk AC, Hartman DJ, et al. Prognostic significance of morphological growth patterns and mitotic index of epithelioid malignant peritoneal mesothelioma. Histopathology. 2016;68:729-37. https://doi.org/1 0.1111/his.12807.

18. Hartman DJ, Borczuk A, Dacic S, Krasinskas A. Reproducibility for histologic parameters in peritoneal mesothelioma. Hum Pathol. 2017;67:54-9. https:// doi.org/10.1016/j.humpath.2017.07.005.

19. Leblay N, Leprêtre F, Le Stang N, et al. BAP1 is altered by copy number loss, mutation, and/or loss of protein expression in more than $70 \%$ of malignant peritoneal mesotheliomas. J Thorac Oncol. 2017;12:724-33. https://doi.org/1 0.1016/j.jtho.2016.12.019.

20. Sheffield BS, Hwang HC, Lee AF, Tet al. BAP1 immunohistochemistry and p16 FISH to separate benign from malignant mesothelial proliferations. Am J Surg Pathol 2015;39:977-982. doi: https://doi.org/10.1097/PAS. 0000000000000394

21. Andrici J, Jung J, Sheen A, et al. Loss of BAP1 expression is very rare in peritoneal and gynecologic serous adenocarcinomas and can be useful in the differential diagnosis with abdominal mesothelioma. Hum Pathol. 2016; 51:9-15. https://doi.org/10.1016/j.humpath.2015.12.012.

22. Borczuk AC, Taub RN, Hesdorffer M, et al. P16 loss and mitotic activity predict poor survival in patients with peritoneal malignant mesothelioma. Clin Cancer Res. 2005;11:3303-8. https://doi.org/10.1158/1078-0432.CCR-04-1884.

23. Krasinskas AM, Bartlett DL, Cieply K, Dacic S. CDKN2A and MTAP deletions in peritoneal mesotheliomas are correlated with loss of p16 protein expression and poor survival. Mod Pathol. 2010;23:531-8. https://doi.org/10.1038/ modpathol.2009.186

24. Pasello G, Zago G, Lunardi F, et al. Malignant pleural mesothelioma immune microenvironment and checkpoint expression: correlation with clinical- pathological features and intratumour heterogeneity over time. Ann Oncol. 2018;29:1258-65. https://doi.org/10.1093/annonc/mdy086.

25. Valmary-Degano $S$, Colpart P, Villeneuve L, et al. Immunohistochemical evaluation of two antibodies against PD-L1 and prognostic significance of PD-L1 expression in epithelioid peritoneal malignant mesothelioma: a RENAPE study. Eur J Surg Oncol. 2017;43:1915-23. https://doi.org/10.1016/j. ejso.2017.05.009.

26. Kusamura S, Torres Mesa PA, Cabras A, et al. The role of Ki- 67 and precytoreduction parameters in selecting diffuse malignant peritoneal mesothelioma (DMPM) patients for Cytoreductive surgery (CRS) and Hyperthermic Intraperitoneal chemotherapy (HIPEC). Ann Surg Oncol. 2016; 23:1468-73. https://doi.org/10.1245/s10434-015-4962-9.

27. Deraco M, Cabras A, Baratti D, Kusamura S. Immunohistochemical evaluation of Minichromosome maintenance protein 7 (MCM7), topoisomerase $\mathrm{lla}$, and $\mathrm{Ki}-67$ in diffuse malignant peritoneal mesothelioma patients using tissue microarray. Ann Surg Oncol. 2015;22:4344-51. https:// doi.org/10.1245/s10434-015-4498-z.

28. Pillai K, Pourgholami MH, Chua TC, Morris DL. Prognostic significance of Ki67 expression in malignant peritoneal mesothelioma. Am J Clin Oncol. 2015;38:388-94. https://doi.org/10.1097/COC.0b013e3182a0e867.

29. Shavelle R, Vavra-Musser K, Lee J, Brooks J. Life expectancy in pleural and peritoneal mesothelioma. Lung Cancer Int. 2017;2017:2782590. https://doi. org/10.1155/2017/2782590

30. Feldman AL, Libutti SK, Pingpank JF, et al. Analysis of factors associated with outcome in patients with malignant peritoneal mesothelioma undergoing surgical debulking and intraperitoneal chemotherapy. J Clin Oncol. 2003: 214560-7. https://doi.org/10.1200/JCO.2003.04.150.

31. Helm JH, Miura JT, Glenn JA, et al. Cytoreductive surgery and hyperthermic intraperitoneal chemotherapy for malignant peritoneal mesothelioma: a systematic review and meta-analysis. Ann Surg Oncol. 2015;22:1686-93. https://doi.org/10.1245/s10434-014-3978-x.

32. García-Fadrique A, Mehta A, Mohamed F, Dayal S, Cecil T, Moran BJ. Clinical presentation, diagnosis, classification and management of peritoneal mesothelioma: a review. J Gastrointest Oncol. 2017;8:915-24. https://doi. org/10.21037/jgo.2017.08.01

33. Yan TD, Deraco M, Elias D, et al. A novel tumor-node-metastasis (TNM) staging system of diffuse malignant peritoneal mesothelioma using outcome analysis of a multi-institutional database*. Cancer. 2011;117:185563. https://doi.org/10.1002/cncr.25640.

\section{Publisher's Note}

Springer Nature remains neutral with regard to jurisdictional claims in published maps and institutional affiliations.

\section{Ready to submit your research? Choose BMC and benefit from:}

- fast, convenient online submission

- thorough peer review by experienced researchers in your field

- rapid publication on acceptance

- support for research data, including large and complex data types

- gold Open Access which fosters wider collaboration and increased citations

- maximum visibility for your research: over $100 \mathrm{M}$ website views per year

At BMC, research is always in progress.

Learn more biomedcentral.com/submission 